Nota

\title{
VISITANTES FLORAIS DE PLANTAS INVASORAS DE ÁREAS COM FRUTEIRAS IRRIGADAS
}

\author{
Lúcia Helena Piedade Kiill ${ }^{1,2 *}$; Francisca Nemaura Pedrosa Haji ${ }^{\text {; }}$ Paulo César Fernandes Lima ${ }^{1}$ \\ ${ }^{1}$ Embrapa Semi-Árido, BR 428, Km 153, Zona Rural, C.P. 23 - CEP: 56300-000 - Petrolina, PE. \\ ${ }^{2}$ Bolsista DCR/CNPq. \\ *Autor correspondente <kiill@cpatsa.embrapa.br>
}

RESUMO : As plantas invasoras afetam a produtividade das fruteiras irrigadas, sendo necessários estudos sobre sua ecologia, principalmente formas de reprodução e associação com insetos. O presente trabalho foi desenvolvido em Petrolina-PE, objetivando classificar os visitantes florais de plantas invasoras em polinizadores e pilhadores, de acordo com o comportamento apresentado. As observações foram feitas de maio a outubro de 1998, em 26 dias não consecutivos, no intervalo das 7 h00 às 16h00. As invasoras foram classificadas em nectaríferas e poliníferas, quando visitadas para retirada exclusiva de néctar ou de pólen; e em mistas, quando visitadas para retirada dos dois recursos. Para a análise de freqüência foi adotado um sistema de notas: anúmero de visitas $>30$, b- entre 10 e 30 e c- $<10$ visitas. Das 24 invasoras observadas, 14 foram consideradas nectaríferas, seis poliníferas e quatro mistas. Entre os visitantes florais foram registradas abelhas (Xylocopa grisescens, $X$. frontalis, Centris aff. perforator, Ptilotrix aff. plumata, Diadasina riparia, Apis mellifera, Trigona spinipes, Eulaema nigrita), borboletas (Ascia monuste, Papilio thoas brasiliensis, Agraulis vanillae) e beijaflores (Phaethornis sp., Chlorostilbon aureoventris). As abelhas apresentaram maior número de espécies (61,5\%) e de visitas, além de agirem como polinizadores em $83 \%$ das invasoras visitadas. As borboletas foram consideradas pilhadores de néctar, participando como polinizadores de Emilia spp. e os beija-flores, considerados polinizadores das invasoras visitadas.

Palavras-chave: polinizador, néctar, pólen, invasoras

\section{FLORAL VISITORS OF WEED PLANTS OF IRRIGATED ORCHARD AREAS}

\begin{abstract}
Weeds can impair yield of irrigated fruit crops, studies about their ecology being needed, especially ways of reproduction and association with insects. This work was carried out in Petrolina, PE, Brazil with the objective of identifying the floral visitors of weeds, and classifying them as pollinators and pollen and/or nectar thieves, according to their behavior. The observations were made between May and October of 1998, in 26 not consecutive days, between 7:00 am and 4:00 pm. The weeds were classified in nectariferous and polliniferous when these were visited exclusively for collection of nectar or pollen, and mixed when these were visited to collect both. A grade system was adopted for the frequency evaluation: a- number of visits $>30, b$ - between 10 to $30, c-<10$. Among the 24 weeds observed, 14 were classified as nectariferous, six as polliniferous and four were considered mixed. Among the floral visitors, the following bees were registered (Xylocopa grisescens, $X$. frontalis, Centris aff. perforator, Ptilotrix aff. plumata, Diadasina riparia, Apis mellifera, Trigona spinipes, Eulaema nigrita), butterflies (Ascia monuste, Papilio thoas brasiliensis, Agraulis vanillae) and hummingbirds (Phaethornis sp., Chlorostilbon aureoventris). The bees were predominant in number of species $(61.5 \%)$ as well as in the frequency of visits. They acted as pollinator agents in $83 \%$ of weeds visited. The butterflies were considered nectar thieves, and they acted as pollinator agents only in the case of the species of Emilia. The hummingbirds acted as pollinators for all visited species.
\end{abstract}

Key words: pollinator, nectar, pollen, weeds

\section{INTRODUÇÃO}

As plantas invasoras se enquadram entre as mais notórias espécies colonizadoras, apresentando características como rápido desenvolvimento, alta plasticidade fenotípica, produção de sementes em grandes quantidades e com alta viabilidade, associadas com eficientes mecanismos de dispersão e dormência, e reprodução por autogamia que favorecem 0 estabelecimento destas espécies em locais continuamente alterados (Baker, 1965, 1974). Por apresentar tais características, são consideradas como um dos fatores que afetam a produtividade agrícola, competindo com as culturas por nutrientes, água e luz, chegando a comprometer de 30 a $40 \%$ da produção (Laca-Buendia et al., 1970; Souza, 1991).

Em levantamentos de plantas invasoras, Blanco $(1972,1978)$ já ressaltava não só os prejuízos causados, como também a escassez de estudos básicos sobre a ecologia dessas plantas no Brasil, mostrando a necessidade de pesquisas que forneçam dados sobre 0 ciclo de desenvolvimento, formas de reprodução e suas 
associações com insetos. Assim, estudos da biologia floral, polinização e reprodução de plantas invasoras são de grande interesse, uma vez que a reprodução por sementes é comum entre essas plantas, constituindo, às vezes, a única forma de dispersão no meio ambiente (Leitão-Filho et al., 1972; Lorenzi, 1982). Neste sentido, é de fundamental importância o conhecimento da morfologia floral, do processo de polinização e dos mecanismos reprodutivos destas plantas, pois, tais informações representam valiosa contribuição para o controle e manejo destas espécies.

O presente trabalho teve como objetivo observar os visitantes florais de plantas invasoras na região de Petrolina-PE, visando identificar os agentes polinizadores destas espécies, bem como o recurso floral por eles forrageado.

\section{MATERIAL E MÉTODOS}

O presente trabalho foi desenvolvido em áreas de fruteiras irrigadas dos Projetos de Irrigação de Bebedouro, Senador Nilo Coelho (Núcleo 5) e Fazenda Boa Esperança, município de Petrolina-PE $\left(09^{\circ} 09^{\prime}\right.$ latitude S, $40^{\circ} 22^{\prime}$ longitude W, $354 \mathrm{~m}$ de altitude), onde observou-se os visitantes florais das espécies invasoras.

As observações foram feitas em parcelas de 30 $\mathrm{m}^{2}$ lançadas aleatoriamente nas áreas escolhidas, totalizando 43 amostras (TABELA 1). Nestas áreas, foram observadas as invasoras que estavam floridas, sendo coletado material botânico para identificação.

De acordo com o recurso floral forrageado, as invasoras foram classificadas em nectaríferas, quando estas eram visitadas para a retirada exclusiva de néctar; poliníferas, quando o pólen era o único recurso floral forrageado pelo visitante; e mistas, quando as flores eram visitadas para a retirada tanto de néctar quanto de pólen.

Os visitantes florais das invasoras foram observados em 26 dias não consecutivos, entre 7h00 e

TABELA 1 - Locais observados com suas respectivas culturas, áreas e número de parcelas avaliados, no município de Petrolina - PE.

\begin{tabular}{llcc}
\hline Local & Cultura & Área & $\begin{array}{c}\text { No. de } \\
\text { parcelas }\end{array}$ \\
\hline Projeto de Irrigação de & Uva & 2,0 & 4 \\
Bebedouro & Manga & 3,0 & 5 \\
& Goiaba & 2,0 & 5 \\
Projeto de Irrigação Senador & Manga & 3,5 & 4 \\
Nilo Coelho - Núcleo 5 & Uva & 3,5 & 4 \\
& Manga & 120,0 & 13 \\
Fazenda Boa Esperança & Uva & 20,0 & 8 \\
\hline Total & & 154,0 & 43 \\
\hline
\end{tabular}

Scientia Agricola, v.57, n.3, p.575-580, jul./set. 2000
$16 \mathrm{~h} 00$, sendo anotados a freqüência, o comportamento de visita e o recurso floral forrageado. O tempo de observação para cada espécie de invasora variou entre 10 e 30 horas, utilizando-se para estas observações no mínimo cinco indivíduos por espécie. Para facilitar a análise da freqüência dos visitantes de cada espécie, foi adotado um sistema de notas : a- para os visitantes que realizaram mais de 30 visitas, b- para os visitantes que realizaram de 10 a 30 visitas, c- para os visitantes que realizaram menos de 10 visitas. De acordo com o comportamento apresentado, os visitantes foram classificados como polinizadores ou pilhadores, segundo Dafni (1992).

Materiais botânicos foram herborizados e enviados ao Herbário do Trópico Semi-Árido (TSAH) para identificação. Os insetos foram coletados, a seco, enviados à Seção de Entomologia da Embrapa Semi-Árido para identificação.

\section{RESULTADOS E DISCUSSÃO}

Foram observadas 24 espécies, 18 gêneros e 9 famílias botânicas, sendo Fabaceae e Malvaceae as que apresentaram o maior número de espécies (TABELA 2). Entre as invasoras, 17 espécies foram comuns a todas as áreas e culturas, enquanto que Canavalia brasiliensis, Centrosema brasilianum e Macroptilium martii só foram observadas nas culturas de manga no Projeto de Irrigação de Bebedouro e na Fazenda Boa Esperança, Phaseolus lathyroides em goiaba e uva no Projeto de Irrigação de Bebedouro, e Crotalaria retusa em manga no Projeto de Irrigação Senador Nilo Coelho.

Entre os visitantes florais foram observadas abelhas (Xylocopa grisescens Lep., X. frontalis Olivier, Centris aff. perforator Sm, Ptilotrix aff. plumata Sm, Diadasina riparia Ducke, Apis mellifera L., Trigona spinipes Fab., Eulaema nigrita Lep.), borboletas (Ascia monuste L., Papilio thoas brasiliensis Rotsch \& Jordan, Agraulis vanillae L.) e beija-flores (Phaethornis sp., Chlorostilbon aureoventris Bourcier \& Mulsant) visitando as plantas invasoras (TABELA 3). Os insetos foram predominantes em número ( $n=11)$, sendo responsáveis por $84,6 \%$ do total de espécies visitantes. Entre os insetos, as abelhas apresentaram o maior número de espécie $(n=8)$, o que representa $61,5 \%$ das espécies visitantes observados.

Entre as abelhas, com exceção de Apis mellifera L., as demais espécies ( $n=7)$ são silvestres. A observação de abelhas nativas visitando flores de invasoras em áreas cultivadas, indica que estas espécies, juntamente com as plantas nativas, fazem parte da dieta alimentar desses himenópteros. Deve-se ressaltar que na estação seca, a vegetação da região reduz drasticamente as fontes alimentares disponíveis, e a presença de plantas invasoras nas áreas irrigadas é um dos poucos recursos disponíveis nesta época do ano, tornando-as fonte obrigatória para estes insetos.

Entre as plantas invasoras, Marsypianthes chamaedrys (Vahl) Kuntze, Raphiodon echinus L. (Lammiaceae), Indigofora hirsuta L., Phaseolus lathyroides 
TABELA 2 - Relação das espécies invasoras observadas em fruteiras irrigadas no município de Petrolina - PE, e seus respectivos locais e culturas de ocorrência.

\begin{tabular}{|c|c|c|c|c|}
\hline \multirow[t]{2}{*}{ Familia } & \multirow[t]{2}{*}{ Nome Científico } & \multirow[t]{2}{*}{ Nome Vulgar } & \multicolumn{2}{|c|}{ Ocorrência } \\
\hline & & & Local $^{*}$ & Cultura \\
\hline \multirow[t]{2}{*}{ Asteraceae } & Emilia sagitata (Valh.) DC. & serralha roxa & geral & todas \\
\hline & Emilia sonchifolia DC. & serralha vermelha & geral & todas \\
\hline \multirow[t]{3}{*}{ Caesalpinaceae } & Senna alata (L.) Roxb. & Canafistula & geral & todas \\
\hline & Senna ocidentalis (L.) Link & Fedegoso & geral & todas \\
\hline & Senna macranthera (Collad) H. S. Irwin \& Barneby & São João & geral & todas \\
\hline Convolvulaceae & Merremia aegyptia (L.) Urb. & Jetirana peluda & geral & todas \\
\hline \multirow[t]{6}{*}{ Fabaceae } & Indig ofora hirsuta L. & Bananinha & geral & todas \\
\hline & Phaseolus lathyroides $\mathrm{L}$. & Feijão de rolinha & 1 & goiaba, uva \\
\hline & Canavalia brasiliensis Benth. & Feijão de boi & 1 e 3 & manga \\
\hline & Centrosema brasilianum (L.) Benth. & Feijão bravo & 1 e 3 & manga \\
\hline & Crotalaria retusa $\mathrm{L}$. & Guiso de cascavel & 2 & manga \\
\hline & Macroptilium martii L. & Orelha de onça & 1 e 3 & manga \\
\hline \multirow[t]{2}{*}{ Lamiaceae } & Marsypianthes chamaedrys (Vahl) Kuntze & Amargosa & geral & todas \\
\hline & Raphiodon echinus L. & Beton & 3 & \\
\hline \multirow[t]{4}{*}{ Malvaceae } & Pavonia cancellata Cav. & Corda de viola 1 & geral & todas \\
\hline & Pavonia humifusa A. St. Hil & Corda de viola 2 & geral & todas \\
\hline & Sida cordifolia L. & Malva grossa & geral & todas \\
\hline & Herissanthia crispa L. & Malva rasteira & geral & todas \\
\hline \multirow[t]{2}{*}{ Passifloraceae } & Passiflora cincinata Mart. & Maracujá de boi & 3 & \\
\hline & Passiflora foetida $\mathrm{L}$. & Maracujá de estalo & geral & todas \\
\hline Rubiaceae & Richardia grandiflora (Cham. \& Schlecht.) Steudel & Ervanço branco & geral & todas \\
\hline \multirow[t]{2}{*}{ Sterculiaceae } & Waltheria indica $\mathrm{L}$. & Malva flor amarela & geral & todas \\
\hline & Waltheria rotundifolia Schrank & Malva prateada & geral & todas \\
\hline Zygophylaceae & Tribulus cistoides $\mathrm{L}$. & Begô & geral & todas \\
\hline
\end{tabular}

*Local : 1- Projeto de Irrigação de Bebedouro, 2- Projeto de Irrigação Senador Nilo Coelho, 3- Fazenda Boa Esperança, geral - em todas as áreas observadas.

L., Canavalia brasiliensis L., Centrosema brasilianum (L.)Benth., Crotalaria retusa L., Macroptilium martii L. (Fabaceae), Richardia grandiflora (Cham. \& Schlecht.) Steudel (Rubiaceae), Waltheria rotundifolia Schrank (Sterculiaceae), Passiflora foetida L., Passiflora cincinata Mart.(Passifloraceae), Emilia sagitata (Valh.) DC. e Emilia sonchifolia DC. (Asteraceae) têm o néctar como o único recurso floral forrageado, e portanto, consideradas nectaríferas. As Fabaceae, Senna alata (L.) Roxb., S. ocidentalis (L.) Link, S. macranthera (Collad) H.S. Irwin \& Barneby, as Malvaceae, Pavonia cancellata, P. humifusa, e Sterculiaceae, Waltheria indica L. têm os grãos de pólen como única fonte alimentar forrageada, sendo então consideradas poliníferas. As espécies Tribulus cistoides $\mathrm{L}$. (Zygophylaceae), Merremia aegyptia (L.) Urb. (Convolvulaceae), Sida cordifolia L. e Herissanthia crispa L. (Malvaceae) têm néctar e pólen forrageados por seus visitantes e foram aqui consideradas como espécies mistas (TABELA 4). Estes resultados corroboram os dados de literatura, onde as plantas invasoras são consideradas como plantas apícolas, fornecedoras tanto de néctar como de pólen (Brandão et al.,1985, 1988; Macedo \& Martins, 1998).

Comparando as plantas invasoras consideradas mistas, notou-se que em Tribulus cistoides e Herissanthia crispa, o mesmo visitante (Apis mellifera) coleta néctar e pólen, enquanto que nas outras duas invasoras os recursos florais são forrageados por espécies diferentes de visitantes. Em Merremia aegyptia observou-se que o néctar foi forrageado por $A$. mellifera, enquanto o pólen foi coletado por Trigona spinipes. Segundo Piedade (1998), esta diferença de comportamento entre as abelhas é atribuída às características morfológicas florais, que selecionam as abelhas com comprimento de língua adequado para ter acesso ao néctar. No caso de $T$. spinipes, estas apresentam comprimento de probóscide inferior ao comprimento do tubo da corola de $M$. aegyptia, impedindo que esta abelha tenha acesso ao néctar, justificando o comportamento de coleta exclusiva de pólen nas flores desta invasora. 
TABELA 3 - Visitantes florais observados nas plantas invasoras em fruteiras, no município de Petrolina- PE.

\begin{tabular}{|c|c|c|c|}
\hline Grupo & Familia & Espécie & Nome Vulgar \\
\hline \multirow[t]{8}{*}{ Himenópteros } & Anthophoridae & Xylocopa grisescens Lep. & Mamangava 1 \\
\hline & & Xylocopa frontalis Olivier & Mamangava 2 \\
\hline & & Centris aff. perforator Sm. & Abelha metálica \\
\hline & & Ptilotrix aff. plumata Sm. & Abelha listrada 1 \\
\hline & & Diadasina riparia Ducke & Abelha listrada 2 \\
\hline & Apidae & Apis mellifera $\mathrm{L}$. & Europa \\
\hline & & Trigona spinipes Fab. & Arapuá \\
\hline & & Eulaema nigrita Lep. & Besouro preto \\
\hline \multirow[t]{3}{*}{ Lepdópteros } & Pieridae & Ascia monuste $\mathrm{L}$. & Borboleta amarela \\
\hline & Papilionidae & Papilio thoas brasiliensis Rotsch \& Jordan & Borboleta marrom \\
\hline & Nymphalidae & Agraulis vanillae $\mathrm{L}$. & Borboleta laranja \\
\hline \multirow[t]{2}{*}{ Beija-flores } & Throchilidae & Chlorostilbon aureoventris Bourcier \& Mulsant & Colibri \\
\hline & Phaetornidae & Phaethornis sp & Beija-flor preto \\
\hline
\end{tabular}

Com relação ao número de plantas invasoras visitadas, verificou-se que há diferenças entre os grupos de visitantes. Das 24 invasoras, nove foram visitadas por Apis mellifera, confirmando que esta abelha é generalista, utilizando todo recurso alimentar que esteja a seu alcance. As flores de Senna alata, S. ocidentalis, S. macranthera, Canavalia brasiliensis, Centrosema brasilianum, Crotalaria retusa, Passiflora foetida e $P$ cincinata foram visitadas por abelhas de grande porte como Xylocopa spp. e Eulaema nigrita Lep. As invasoras das famílias Malvaceae e Convolvulaceae foram visitadas preferencialmente por abelhas pequenas como Trigona spinipes, Ptilotrix aff. plumata Sm. e Diadasina riparia Duke. Estas duas últimas foram observadas somente em duas invasoras, sendo ambas do gênero Pavonia. Tais informações indicam que há preferência entre as abelhas por determinadas flores.

As flores de Indigofora hirsuta, Raphiodon echinus, Richardia grandiflora, Centrosena brasilianum, Macroptilium martii, Waltheria rotundifolia, Emilia sagitata e E. sonchifolia foram visitadas preferencialmente pelas borboletas. Destas oito invasoras, somente Emilia spp. tiveram suas flores visitadas exclusivamente por Ascia monuste L. e Agraulis vanillae L. As flores de Marsypianthes chamaedrys, Phaseolus lathyroides, Canavalia brasiliensis e Sida cordifolia foram visitadas por beija-flores. Entre estes visitantes, nota-se que o Phaethornis $\mathrm{sp}$ visitou as flores de C. brasiliensis, enquanto Chlorostilbon aureoventris Bourcier \& Mulsant. visitou flores das demais espécies. No primeiro caso, esta preferência por $C$. brasiliensis pode estar associado ao posicionamento das flores, que se encontravam sobre as mangueiras (Manguifera indica - Anacardiaceae) ou sobre outras invasoras arbustivas, ficando numa posição elevada, que então teria atraído este visitante. As outras invasoras, por serem rasteiras ou herbáceas teriam atraído C. aureoventris, que é um beija-flor de porte pequeno e de vôos mais baixos.
Quanto ao comportamento de visita e recurso floral forrageado, houve diferenças entre os visitantes. Entre as abelhas, o comportamento de coleta de néctar foi semelhante, porém para a coleta de pólen diferenças foram registradas. Na coleta de néctar, dependendo da espécie visitada, as abelhas pousavam sobre a corola de uma flor ou sobre a inflorescência e, posteriormente, introduziam a probóscide no interior da corola, tocando anteras e estigma com as regiões dorsal (polinização nototríbica) ou ventral (polinização esternotríbica) do corpo, onde os grãos de pólen ficavam depositados. Apis mellifera, Xylocopa spp., Eulaema nigrita e Centis aff. perforator $\mathrm{Sm}$. apresentaram este comportamento em suas visitas às flores de 13 espécies de invasoras (TABELA 4), onde são consideradas como agentes polinizadores. Para Passiflora foetida, as abelhas $A$. mellifera e E. nigrita são consideradas como pilhadores de néctar, pois, não contatam as estruturas reprodutivas durante a coleta deste recurso.

$\mathrm{Na}$ coleta de pólen, dois comportamentos foram observados. Xylocopa spp., em suas visitas às flores das espécies de Senna, apresentaram comportamento de coleta de pólen por vibração (Buchmann, 1983), pousando sobre as estruturas reprodutivas e, com o auxílio das patas e contrações do tórax e abdômen, vibravam as anteras para a retirada dos grãos de pólen, ficando estes depositados na região ventral do corpo dos insetos (polinização esternotríbica). Este comportamento é relatado para outras espécies de Fabaceae, mostrando a coevolução entre flores e seus agentes polinizadores (Faegri \& Pijl, 1980; Buchmann, 1983). As abelhas Apis mellifera e Trigona spinipes em suas coletas de pólen pousavam sobre a corola ou diretamente sobre as anteras e, com o auxílio das patas e peças bucais retiravam os grãos de pólen das anteras, ficando o pólen depositado na região da cabeça e parte ventral do corpo dos insetos. 
TABELA 4 - Plantas invasoras com seus respectivos visitantes florais e recursos florais forrageados. Petrolina - PE.

\begin{tabular}{|c|c|c|c|c|c|c|c|c|c|c|c|c|c|}
\hline \multirow{3}{*}{$\begin{array}{l}\text { Plantas } \\
\text { Invasoras }\end{array}$} & \multicolumn{13}{|c|}{ Visitantes Florais } \\
\hline & \multicolumn{8}{|c|}{ Abelhas } & \multicolumn{3}{|c|}{ Borboletas } & \multicolumn{2}{|c|}{ Beija-flores } \\
\hline & $\begin{array}{c}A . \\
\text { mellifera }\end{array}$ & $\begin{array}{c}X . \\
\text { grisescens }\end{array}$ & $\begin{array}{c}X . \\
\text { frontalis }\end{array}$ & $\begin{array}{c}T . \\
\text { spinipes }\end{array}$ & $\begin{array}{c}E . \\
\text { nigrita }\end{array}$ & $\begin{array}{c}\text { C. aff. } \\
\text { perforator }\end{array}$ & $\begin{array}{c}\text { P. aff. } \\
\text { plumata }\end{array}$ & $\begin{array}{c}D . \\
\text { riparia }\end{array}$ & $\begin{array}{c}A . \\
\text { monuste }\end{array}$ & $\begin{array}{c}P . \text { thoas } \\
\text { brasiliensis }\end{array}$ & $\begin{array}{c}A . \\
\text { vanillae a }\end{array}$ & $\begin{array}{c}C . \\
\text { aureoventris }\end{array}$ & $\begin{array}{c}\text { Phaethornis } \\
s p \\
\end{array}$ \\
\hline E. sagitata & & & & & & & & & $b, n, p o$ & & & & \\
\hline $\begin{array}{l}\text { E. } \\
\text { sonchifolia }\end{array}$ & & & & & & & & & $\mathrm{b}, \mathrm{n}, \mathrm{po}$ & & $c, n, p o$ & & \\
\hline M. aegyptia & $\mathrm{a}, \mathrm{n}, \mathrm{po}$ & & & $a, p, p o$ & & & & & & & & & \\
\hline 1. hirsuta & $\mathrm{a}, \mathrm{n}, \mathrm{po}$ & & & & & & & & $\mathrm{b}, \mathrm{n}, \mathrm{pi}$ & & $\mathrm{c}, \mathrm{n}, \mathrm{pi}$ & & \\
\hline S. alata & & $a, p, p o$ & $a, p, p o$ & & & & & & & & & & \\
\hline $\begin{array}{l}\text { S. } \\
\text { ocidentalis }\end{array}$ & & $a, p, p o$ & $a, p, p o$ & & & & & & & & & & \\
\hline $\begin{array}{l}\text { S. } \\
\text { macranthera }\end{array}$ & & $a, p, p o$ & $a, p, p o$ & & & & & & & & & & \\
\hline $\begin{array}{l}P . \\
\text { lathyroides }\end{array}$ & & & & & & & & & & & & c, n;po & \\
\hline $\begin{array}{l}\text { C. } \\
\text { brasiliensis }\end{array}$ & & $\mathrm{a}, \mathrm{n}, \mathrm{po}$ & $a, n, p o$ & & $\mathrm{a}, \mathrm{n}, \mathrm{po}$ & & & & & & & & $b, n, p o$ \\
\hline $\begin{array}{l}\text { C. } \\
\text { brasilianum }\end{array}$ & & $\mathrm{a}, \mathrm{n}, \mathrm{po}$ & $b, n, p o$ & & $\mathrm{a}, \mathrm{n}, \mathrm{po}$ & $\mathrm{a}, \mathrm{n}, \mathrm{po}$ & & & $\mathrm{c}, \mathrm{n}, \mathrm{pi}$ & & & & \\
\hline C. retusa & & $\mathrm{a}, \mathrm{n}, \mathrm{po}$ & $b, n, p o$ & & & & & & & & & & \\
\hline M. martii & $\mathrm{a}, \mathrm{n}, \mathrm{po}$ & & & & & & & & $\mathrm{b}, \mathrm{n}, \mathrm{pi}$ & & & & \\
\hline $\begin{array}{l}\text { M. } \\
\text { chamaedrys }\end{array}$ & & & & & & & & & & & & $c, n, p o$ & \\
\hline R. echinus & $\mathrm{a}, \mathrm{n}, \mathrm{po}$ & & & & & & & & $\mathrm{b}, \mathrm{n}, \mathrm{pi}$ & & $\mathrm{c}, \mathrm{n}, \mathrm{pi}$ & & \\
\hline P. cancellata & & & & & & & $a, p, p o$ & $a, p, p o$ & & & & & \\
\hline P. humifusa & & & & & & & $a, p, p o$ & $a, p, p o$ & & & & & \\
\hline S. cordifolia & & & & $b, p, p o$ & & & & & & & & $c, n, p o$ & \\
\hline H. crispa & $a, n / p, p o$ & & & $c, p, p o$ & & & & & & & & & \\
\hline P. foetida & $\mathrm{a}, \mathrm{n}, \mathrm{pi}$ & $b, n, p o$ & $b, n, p o$ & & $\mathrm{c}, \mathrm{n}, \mathrm{pi}$ & & & & & & & & \\
\hline P. cincinata & & $\mathrm{a}, \mathrm{n}, \mathrm{po}$ & $\mathrm{a}, \mathrm{n}, \mathrm{po}$ & & & & & & & & & & \\
\hline $\begin{array}{l}R . \\
\text { grandiflora }\end{array}$ & $\mathrm{a}, \mathrm{n}, \mathrm{po}$ & & & & & & & & & $\mathrm{c}, \mathrm{n}, \mathrm{pi}$ & $c, n, p i$ & & \\
\hline W. indica & & & & $b, p, p o$ & & & & & & & & & \\
\hline $\begin{array}{l}W . \\
\text { rotundifolia }\end{array}$ & $\mathrm{a}, \mathrm{n}, \mathrm{po}$ & $b, n, p o$ & & & & & & & & & $\mathrm{c}, \mathrm{n}, \mathrm{pi}$ & & \\
\hline
\end{tabular}

Legenda : a- >30 visitas, b- entre 10 e 30 visitas, c- < 10 visitas, $n$ - néctar, $p$ - pólen, $n / p$ - néctar e pólen, po- polinizador, pi- pilhador

De modo geral, as abelhas participam como agentes polinizadores de 20 das 24 invasoras observadas, indicando que estes himenópteros desempenham importante papel na polinização de plantas invasoras. As abelhas têm papel relevante na polinização dos vegetais (Amaral, 1968, 1971, 1972; Amaral \& Alves, 1976; Weise, 1975), sendo relatada com um dos principais agentes para a comunidade da caatinga (Machado, 1990; Piedade, 1998).

As borboletas, apresentaram comportamento semelhante, forrageando somente néctar das flores. Para a coleta deste recurso, pousavam sobre as flores ou inflorescência, introduziam a espirotromba no interior da corola, e realizando curtos deslocamentos para frente e para trás, sugavam o néctar. Ao realizar tais deslocamentos, tocavam as estruturas reprodutivas com a cabeça e/ou peças bucais, promovendo a polinização. Das oito espécies visitadas, as borboletas agem como polinizadores em Emilia spp.(2 espécies) e como pilhadores de néctar nas demais plantas. Beija-flores, foram observados visitando as flores de Marsypianthes chamaedrys, Phaseolus lathyroides, Canavalia brasiliensis e Sida cordifolia. Ambos apresentaram comportamento de visita semelhante, com o adejamento a uma determinada flor, introduzindo parte do bico no interior da corola, sugando o néctar e contatando as estruturas reprodutivas com a parte apical do bico. As flores visitadas por estes pássaros apresentam coloração e forma que não se enquadram no padrão típico das flores ornitófilas, que se caracterizam por serem tubulares e de coloração vermelha (Percival, 1969; Faegri \& Pijl, 1980). As visitas de beija-flores a estas invasoras mostraram que estes são generalistas, e para manter sua dieta alimentar, visitam flores que não se enquadram no padrão ornitófilo.

Quanto ao número de visitas, as abelhas foram as mais freqüentes, geralmente apresentando número de visitas superior a 30 . As borboletas e os beija-flores apresentaram freqüências menores, com números de visitas inferior a 30. Esta diferença vem reforçar a importância destes insetos na polinização de invasoras, e segundo Brandão et al. (1988), o êxito deste grupo como polinizadores deve-se ao fato de serem insetos sociais, 
numerosos, que apresentam instinto gregário, visitando as flores não só para satisfazer suas necessidades alimentícias individuais, mas também para armazenar o alimento excedente que será utilizado na alimentação de outros indivíduos da colmeia.

\section{CONCLUSÕES}

As abelhas agem como polinizadores em 20 das 24 espécies observadas. As borboletas são consideradas pilhadores de néctar, participando como polinizadores de Emilia sagitata e E. sonchifolia, e os beija-flores agem como polinizadores em todas as invasoras visitadas. As plantas invasoras podem ser consideradas fonte alternativa de néctar e pólen e, devido a sua grande adaptabilidade às condições edafoclimáticas, são passíveis de serem utilizadas como plantas apícolas.

\section{REFERÊNCIAS BIBLIOGRÁFICAS}

AMARAL, E. Polinização do algodoeiro pelas abelhas Piracicaba: ESALQ, 1968.1p.

AMARAL, E. As abelhas na polinização do pepino. Piracicaba: ESALQ, 1971. 3p.

AMARAL, E. Produção de café na ausência e na presença de insetos polinizadores. In : CONGRESSO BRASILEIRO DE APICULTURA, 2., Sete Lagoas, 1972. Anais. Sete Lagoas: IPEACO, 1972. p.59-67.

AMARAL, E.; ALVES, S.B. Estudos sobre a polinização da mangueira. Revista Brasileira de Agricultura, v.51, p.251-252, 1976.

BAKER, H.G. Charcteristics and modes of origin of weeds. In: BAKER, H.G.; STEBBINS, G.L.(Ed.) The genetics of colonizing species. New York: Academic Press, 1965. p.147-172.

BAKER, H.G. The evolution of weeds. Annual Review of Ecology and Systematics, v.5, p.1-24, 1974.

BLANCO, H.G. A importância dos estudos ecológicos nos programas de controle de plantas daninhas. O Biológico, v.38, p.343-350, 1972.

BLANCO, H.G. Catálogo das espécies de mato infestantes de áreas cultivadas no Brasil - Família das campainhas (Convolvulaceae). O Biológico, v.44, p.259-278, 1978.
BRANDÃO, M.; LACA-BUENDIA, J.P.; GAVINALES, M.L.; ZURLO, M.A.; CUNHA, L.H.S.; CARDOSO, C. Novos enfoques para plantas consideradas daninhas. Informe Agropecuário, v.11, p.3-12, 1985.

BRANDÃO, M.; LACA-BUENDIA, J.P.; GAVINALES, M.L.; CASTELOIS, B.C.R.J.; CUNHA, L.H.S. Plantas daninhas como possibilidades apícolas. Informe Agropecuário, v.13, p.3-17, 1988.

BUCHMANN, S.L. Buzz pollination in angiosperms. In: JONE, E.C.; LITTLE R.J. (Ed.) Handbook of experimental pollination biology. New York: Scientific and Academic, 1983. p.73-113

DAFNI, A. Pollination ecology. New York: Oxford University Press, 1992. 250p.

FAEGRI, K.; PIJL, L. van der. Principles of pollination ecology. 3.ed. London: Pergamon Press, 1980. 244p.

LACA-BUENDIA, J.P. del C.; PURCINO, A.A.C.; PENNA J.C.V.; FERREIRO, L. Período crítico de competição entre comunidades de plantas daninhas e o algodão (Gossypium hirsutum L.) no Estado de Minas Gerais. Planta Daninha, v.2, p.89-95, 1970.

LEITÃO-FILHO, H.; ARANHA, C.; BACCHI, O. Plantas invasoras de culturas no Estado de São Paulo. Campinas: Instituto Campineiro de Ensino Agrícola, 1972. v.1, 291p.

LORENZI, H.J. Plantas daninhas do Brasil: terrestres, aquáticas, parasitas, tóxicas e medicinais. Nova Odessa: $\mathrm{H}$. Lorenzi, 1982. 425p.

MACEDO, J.F.; MARTINS, R.P. Potencial da erva daninha Waltheria americana (Sterculiaceae) no manejo integrado de pragas e polinizadores: visitas de abelhas e vespas. Anais da Sociedade Entomológica do Brasil, v.27, p.29-40, 1998.

MACHADO, I.C.S. Biologia floral de espécies da caatinga no município de Alagoinha (PE). Campinas, 1990. 145p. Tese (Doutorado) - Universidade Estadual de Campinas.

PERCIVAL, M.S. Floral biology. London: Pergamon Press, 1969. $243 p$.

PIEDADE, L.H. Biologia da polinização e reprodutiva de sete espécies de Convolvulaceae da caatinga do Sertão de Pernambuco. Campinas, 1998. 105p. Tese (Doutorado)Universidade Estadual de Campinas.

SOUZA, I.F. Controle biológico de plantas daninhas. Informe Agropecuário, v.15, p.77-82, 1991.

WEISE, H. Ensaio de polinização entomófila com abelhas em macieiras: Projeto apicultura. Florianópolis: Secretaria da Agricultura do Estado de Santa Catarina, 1975. 15p.

$\overline{\text { Recebido em } 31.08 .99}$ 